\title{
Supplement zum 7. HeilberufeSCIENCE-Symposium auf dem 5. Interprofessionellen Gesundheitskongress in Dresden
}

\author{
J. Klewer ${ }^{1}$ A. Tauchert ${ }^{2}$
}

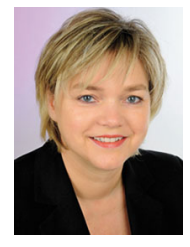

Andrea Tauchert

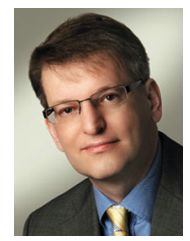

Jörg Klewer

Nach der erfolgreichen Veranstaltung im vergangenen Jahr findet dieses Jahr das mittlerweile 7. HeilberufeSCIENCESymposium wieder in Dresden statt. Wie in den Vorjahren bilden die wissenschaftlichen Postersessions sowie die Vorträge eine Einheit, die zum fachlichen Austausch und zur Diskussion einlädt - und gleichzeitig den Theorie-PraxisTransfer fördert. Insbesondere die zentral im Kongresszentrum platzierten Postersessions fanden im vergangenen Jahr große Aufmerksamkeit, woraus sich zahlreiche angeregte Gespräche entwickelten. An diese gute Tradition möchte

J. Klewer

joklewer@t-online.de

1 Fakultät, Westsächsische Hochschule Zwickau, Scheffelstr. 39, Zwickau, Deutschland

2 Kongressorganisation, Berlin, Deutschland das 7. HeilberufeSCIENCE-Symposium natürlich wieder anknüpfen.

Dieses HeilberufeSCIENCE-Supplement beinhaltet alle Abstracts der Vorträge und Poster des 7. HeilberufeSCIENCE-Symposiums in einer zitierfähigen Form. Alle Abstracts können Sie nach dem Kongress jederzeit auch von den HeilberufeSCIENCE-Seiten im Portal SpringerLink abrufen. Dort finden Sie auch die regulären Ausgaben von HeilberufeSCIENCE, Informationen zur Einreichung von Beiträgen, zum Blinded-Peer-Review-Verfahren und zu den Listungen, u. a. in CINAHL ${ }^{\circledR}$ Complete.

An dieser Stelle möchten wir Ihnen die Lektüre dieses Supplements empfehlen - sei es zur Vorbereitung auf das 7. HeilberufeSCIENCE-Symposium oder um im Nachgang einzelne Inhalte noch einmal zu vertiefen! Natürlich würden wir uns freuen, wenn wir Sie am 28. und 29. April auf dem 5. Interprofessionellen Gesundheitskongress in Dresden begrüßen dürfen.

Ihre

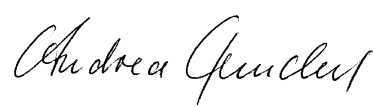

Andrea Tauchert

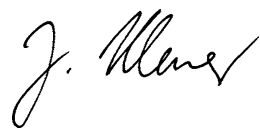

Jörg Klewer 